\title{
Trajectory of a road vehicle during road maintenance
}

\author{
Darina Stachová ${ }^{1 *}$ \\ ${ }^{1}$ Department of Technical Sciences and Informatics, Faculty of Security Engineering, University of \\ Žilina, Univerzitná 8215/1, 01026 Žilina, Slovakia
}

\begin{abstract}
Consider a vehicle moving on a road whose usage over time creates an uneven surface on the road. Road unevenness that we encounter on surface communications often arises as a consequence of dynamical effects of moving vehicles, of weather changes, and due to road construction works. This article concerns with mathematical modeling of the trajectory of a road vehicle moving on such a surface during the course of road maintenance.
\end{abstract}

\section{Introduction}

Consider a vehicle moving on a road whose usage over time creates an uneven surface on the road. The problem we want to focus on here is mathematical modeling of the trajectory that this vehicle traverses while moving on such a road. For simplicity, we identify the motion of the vehicle with the motion of the center axis of a moving wheel [1]. Unevenness that we encounter on surface communications (besides intentionally positioned speed bumps) are typically a consequence of dynamical effects of moving vehicles, due to changes in weather, and due to reconstruction of the road surface. A vehicle moving on a road must often adjust its speed based on the state and shape of the road. This fact is a reason to focus on modeling of the vehicle's trajectory on a road with damaged surface [2]. In particular, driving on such a road is safe and economical, if the trajectory constitutes a smooth curve, i.e. a curve without singular points. This, of course, assumes that the road

\section{Computational model of a road}

For the purposes of this contribution, let us consider a road to be a surface communication with hard and uneven surface. Mathematical description of a computational model of such a road then reduces to a mathematical model of unevenness of the trajectory of a vehicle [3].

In the course of a road reconstruction, the following three situations can occur:

1. vehicle driving through a right-angle asphalt cutout after the removal of the asphalt layer from the road surface,

\footnotetext{
* Corresponding author: Darina.Stachova@,fbi.uniza.sk
} 
2. vehicle driving through a trapezoidal asphalt cutout after the removal of the asphalt layer from the road surface,

3. vehicle driving through a step over a steel board positioned to cover the terrain during excavation works.

We shall assume that the wheels of the vehicle undergo no deformation during the motion and maintain their circular shape throughout. This is a reasonable assumption to make, since in practice the changes of wheel shape are negligible (thanks to modern technologies) when compared to the changes in road surface that we consider here. We shall therefore consider the problems that arise when rolling a circular wheel on a road with damaged surface [4].

\subsection{Road with a right-angle cutout}

Assume that the surface of the road has the shape of a right-angle cutout as illustrated in Fig. 1, where we denote by $l$ the length of the cutout, by $h$ the depth of the cutout, and by $r$ the radius of the moving wheel.

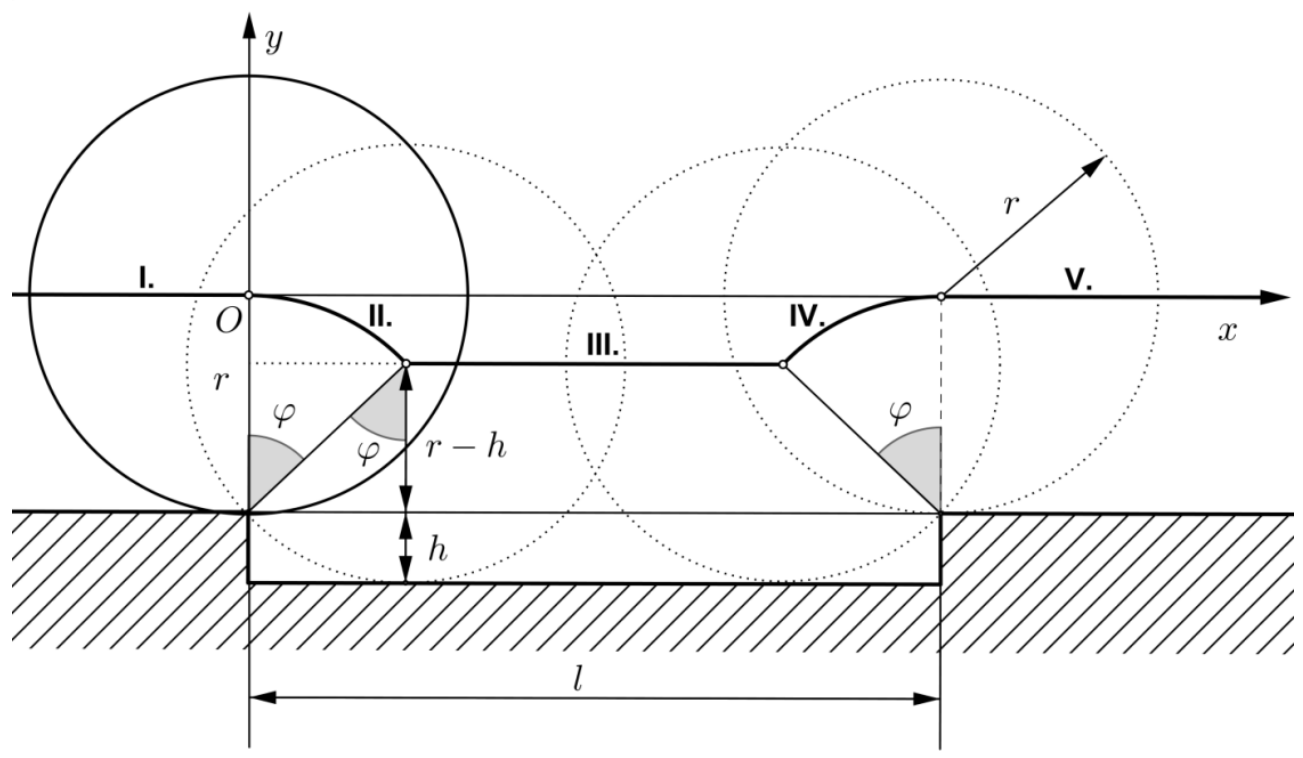

Fig. 1. Road with a right-angle cutout.

The shape of the road surface is described by a function that is not differentiable everywhere. The jumps in the function value at the beginning and the end of the cutout constitute from the mathematical perspective point singularities, which are relevant also for answering non-mathematical questions. The (axis of the) wheel when driving through the cutout does not precisely copy the shape of the cutout, since the motion of the wheel axis in the vertical direction is caused by kinematic excitation of the suspended mass of the vehicle. The axis traverses a trajectory consisting of line segments and circular arcs denoted in Fig. 1 as I., II., III., IV., and V. When moving across the two ends of the cutout, the axis moves along the circular arcs II. and IV., which can be represented in the $(O, x, x)$ coordinate system in different ways, for instance, in the explicit form. Since the angle $\varphi$ is small compared to the depth $h$, we can use polynomial approximations (this choice is also meaningful due to the technical and practical use of such approximations) [5]. Since the 
circular arcs are short, we can sufficiently accurately approximate them with section of parabolas $y(x)=\frac{-x^{2}}{2 p}$, resp. $y(x)=\frac{-(x-l)^{2}}{2 p}$, where $p$ is the parameter to be determined.

The complete description of the trajectory is then as follows.

I. $\quad y(x)=0$

$$
x \in(-\infty ; 0)
$$

II. $\quad y(x)=\frac{-x^{2}}{2 r-h}$

$$
x \in\left(0 ; \sqrt{2 r h-h^{2}}\right)
$$

III. $y(x)=-h$

$$
x \in\left(\sqrt{2 r h-h^{2}} ; l-\sqrt{2 r h-h^{2}}\right)
$$

IV. $\quad y(x)=\frac{-(x-l)^{2}}{2 r-h}$

$$
x \in\left(l-\sqrt{2 r h-h^{2}} ; l\right)
$$

V. $y(x)=0$

$x \in(l ; \infty)$

Critical points of this trajectory occur when transitioning from segment I. to II, and from IV. to V. In these points the vehicle has a two-point contact with the surface, which creates a singularity in the trajectory.

\subsection{Road with a trapezoidal cutout}

Assume that the road surface has a shape of a trapezoidal cutout. This is illustrated in Fig. 2. The size of the depth angle $\varphi$ determines the shape and the components of the wheel's trajectory.

\subsubsection{Road with a trapezoidal cutout of type $A$}

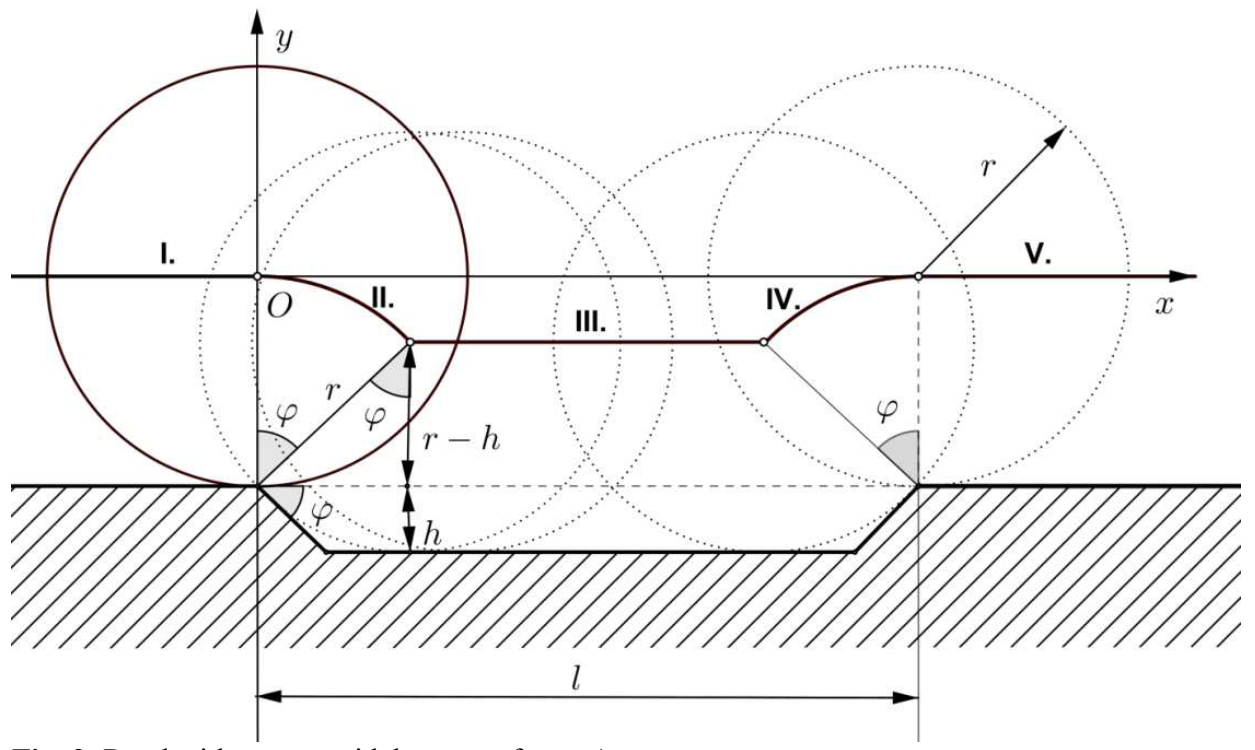

Fig. 2. Road with a trapezoidal cutout of type A. 
If the angle $\varphi$ satisfies: $\arccos \frac{r-h}{r} \leq \varphi \leq \frac{\pi}{2}$, then the two-point contact of the wheel with the surface only occurs at the beginning and the end of the cutout. The mathematical description of the trajectory is identical to Case 1, i.e. it is again composed of five segments described by the equations (1) - (5) listed above.

\subsubsection{Road with a trapezoidal cutout of type $B$}

If the angle $\varphi$ satisfies: $\varphi<\arccos \frac{r-h}{r}$, as illustrated in Fig. 3, then again a two-point contact of the wheel with the surface occurs in two places, however, this time not at the beginning and the end of the cutout. The mathematical description of the trajectory now consists of seven segments and can be expressed as follows.

I. $y(x)=0$

$x \in(-\infty ; 0)$

II. $y(x)=\frac{-1}{r(1+\cos \varphi)} x^{2}$

III. $y(x)=(-\operatorname{tg} \varphi) x+r\left(\frac{1}{\cos \varphi}-1\right)$

$x \in\left(r \cdot \sin \varphi ; \frac{r}{\sin \varphi}+\frac{h-r}{\operatorname{tg} \varphi}\right)$

IV. $y(x)=-h$

$x \in\left(\frac{r}{\sin \varphi}+\frac{h-r}{\operatorname{tg} \varphi} ; l-\frac{r}{\sin \varphi}-\frac{h-r}{\operatorname{tg} \varphi}\right)$

V. $y(x)=(\operatorname{tg} \varphi) \cdot(x-l)+r\left(\frac{1}{\cos \varphi}-1\right)$

$x \in\left(l-\frac{r}{\sin \varphi}-\frac{h-r}{\operatorname{tg} \varphi} ; l-r \cdot \sin \varphi\right)$

VI. $y(x)=\frac{-1}{r(1+\cos \varphi)}(x-l)^{2}$

$x \in(l-r \cdot \sin \varphi ; l)$

VII. $y(x)=0$

$x \in(l ; \infty)$

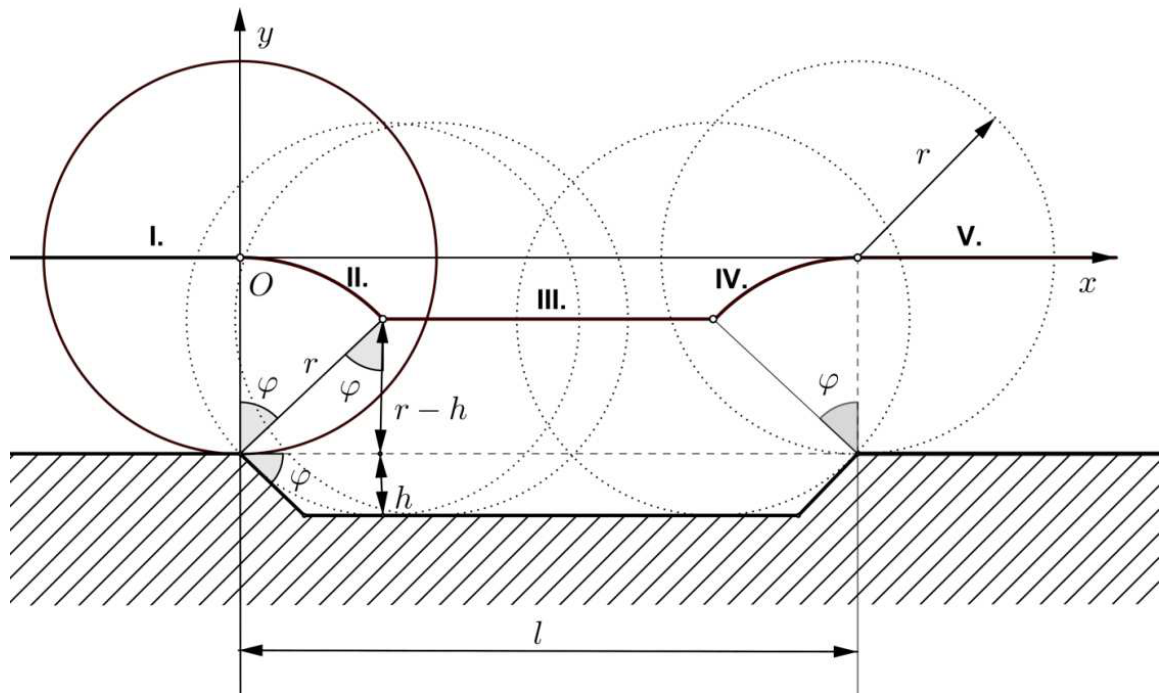

Fig. 3. Road with a trapezoidal cutout of type B. 


\subsection{Rectangular step}

In the last case, we look at the road surface in the shape of a rectangular terrain step, as illustrated in Fig. 4.

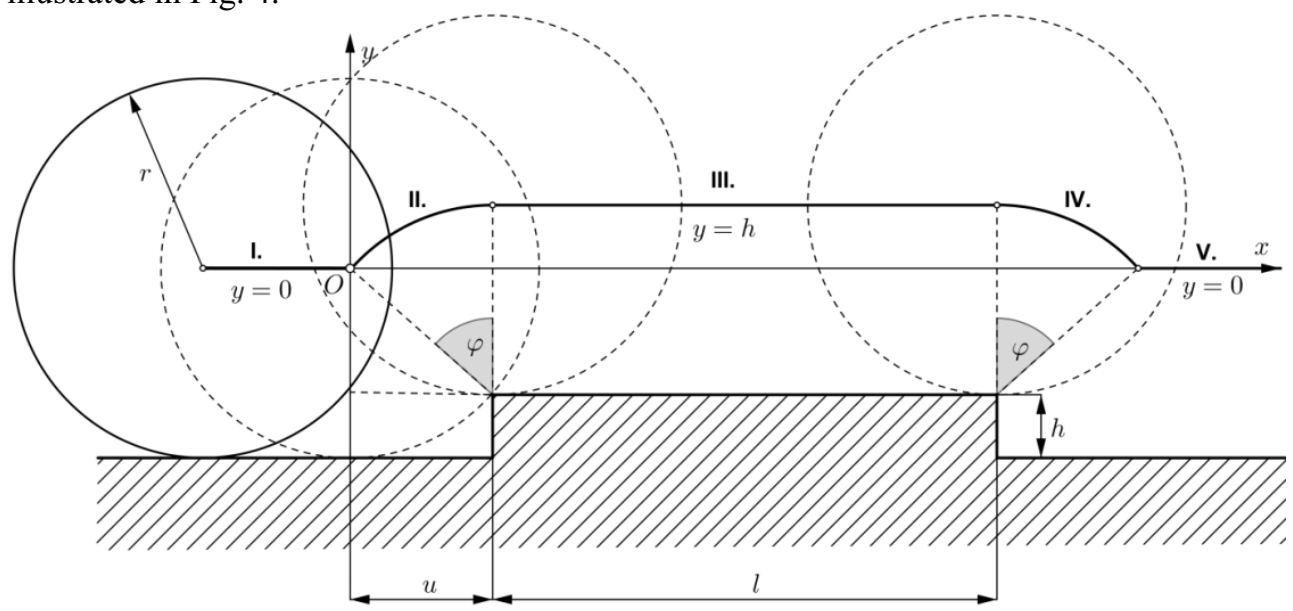

Fig. 4. Road surface with a terrain step.

During excavation works in the course of construction on the road, the resulting trenches are often covered by steel plates to allow vehicles to continue using the road. The surface of this road can be then modeled using a rectangular step in the coordinate system $(O, x, y)$, as shown in Fig. 4, where we denote by $l$ the length of the step, by $h$ the height of the step, by $u$ the distance of the step from the $y$ axis, and by $r$ the radius of the moving wheel. The axis of the wheel traverses a trajectory comprised of five parts. The singular points are this time found before and after the step. The jumps in the function value at the beginning and the end of the step constitute in the trajectory of the wheel the motion along circular arcs II. and IV. by turning the wheel by angle $\varphi$. Therefore $u=r \cdot \sin \varphi$ and $|r-h|=r \cdot \cos \varphi$. The two arcs can be expressed in the Cartesian coordinates explicitly as follows.
II. $\quad(x-u)^{2}+(y-h+r)^{2}-r^{2}=0$
for $\quad x \in\langle 0 ; u\rangle$
IV. $(x-l-u)^{2}+(y-h+r)^{2}-r^{2}=0$
for $\quad x \in\langle l+u ; l+2 u\rangle$

Since the arcs are short (because $h$ is relatively small compared to $r$ ), we approximate the arcs using parabolas of second order as follows.
II. $y=h-\frac{h}{u^{2}}(x-u)^{2}$
$x \in\langle 0 ; u\rangle$
IV. $y=h-\frac{h}{u^{2}}(x-l-u)^{2}$
$x \in\langle l+u ; l+2 u\rangle$

\section{Conclusions}

The vehicle-road interaction is a current problem that is a focus of attention of engineers around the world. The best way to solve these types of problems is a combination of numerical and experimental methods. Numerical methods today provide an effective tool 
for solving these problems using the state-of-the-art computers, which allow solving these problems practically in real time.

The search for and removal of singular points on the road is monitored for ensuring safe ride on damaged roads. The existence of these points causes the shape of the trajectory of the vehicle to not agree with the shape of the road surface in places where the road is damaged. In general, solving some of the problems is not simple. In particular, the problems become difficult in cases when the wheel loses contact with the road surface. This is, on the one hand, due to the fact that at the points of the loss of contact the character of vibrations changes from forced to free, and on the other hand, the points when the contact is restored cause impact between the wheel and the surface. In particular, to explain the conditions at the moment of the impact requires a separate complex analysis.

The results of numerical and experimental analyses are applied in the design of road vehicles as well as in the road design, and to improve comfort of passenger. Acquired results are also used when designing optimal parameters of the road when it comes to its reliability, longevity, and maintenance costs.

This work was produced as part of the grant project VEGA SR 1/0005/16.

\section{References}

1. J. Melcer, D. Stachová, Proceedings of the $2^{\text {nd }}$ International Conference Dynamics of Civil Engineering and Transport Structures and Wind Engineering, DYN-WIND 2017, 178-181 (2003)

2. J. Melcer, Maintenance and its impact on roads and highways, Seminar with international participation, Žilina, Slovakia, 55-58 (1999)

3. J. Melcer, Dynamic calculations of highway bridges, (EDIS, Žilina, 1997, in Slovak)

4. D. Stachová, Proceedings of the Symposium on Computer Geometry SCG '2006, 15, 130-135 (2006)

5. D. Stachová, Proceedings of the Symposium on Computer Geometry SCG '2008, 17, 87-90 (2008) 